\title{
Transient scaling and resurgence of chimera states in networks of Boolean phase oscillators
}

\author{
David P. Rosin, ${ }^{1,2}$ Damien Rontani, ${ }^{1,3}$ Nicholas D. Haynes, ${ }^{1}$ Eckehard Schöll, ${ }^{2}$ and Daniel J. Gauthier ${ }^{1}$ \\ ${ }^{1}$ Department of Physics, Duke University, 120 Science Drive, Durham, North Carolina 27708, USA \\ ${ }^{2}$ Institut für Theoretische Physik, Technische Universität Berlin, Hardenbergstraße 36, Berlin D-10623, Germany \\ ${ }^{3}$ Supélec, OPTEL Research Group and LMOPS EA-4423, 2 Rue Edouard Belin, Metz F-57070, France
}

(Received 8 May 2014; published 30 September 2014)

\begin{abstract}
We study networks of nonlocally coupled electronic oscillators that can be described approximately by a Kuramoto-like model. The experimental networks show long complex transients from random initial conditions on the route to network synchronization. The transients display complex behaviors, including resurgence of chimera states, which are network dynamics where order and disorder coexists. The spatial domain of the chimera state moves around the network and alternates with desynchronized dynamics. The fast time scale of our oscillators (on the order of $100 \mathrm{~ns}$ ) allows us to study the scaling of the transient time of large networks of more than a hundred nodes, which has not yet been confirmed previously in an experiment and could potentially be important in many natural networks. We find that the average transient time increases exponentially with the network size and can be modeled as a Poisson process in experiment and simulation. This exponential scaling is a result of a synchronization rate that follows a power law of the phase-space volume.
\end{abstract}

DOI: 10.1103/PhysRevE.90.030902

PACS number(s): 05.45.Xt, 64.60.aq, 84.30.Ng

As discovered recently, the dynamical state of networks can show a surprising behavior called chimeras, where network nodes split into coexisting domains of entirely different dynamics, such as synchronized and unsynchronized dynamics [1-7]. Chimera states have possible applications to brain activity patterns, cardiac fibrillation, and social systems [8]. Recently, chimera states have been identified theoretically as long chaotic transients towards synchrony for finite-size networks, scaling exponentially with the system size [9]. Such an exponential scaling of the transient with the system size is called a supertransient in extended systems, but is not commonly known to appear in networks.

Predicting the time scale for the transition to synchronization is crucial for technological applications, such as cascading failure in power grids and turbulent flows in pipes [10,11]. Furthermore, it is of great importance for biological systems, such as ecological and neural systems [12,13]. Transient scaling is especially important in networks because network structures dominate many natural and engineered systems [14], but has not yet been shown experimentally. For example, for networks displaying chimera states, slow characteristic time scales have previously prevented the measurement of transient scaling [15-20]. Even in theoretical studies, this scaling could only be verified in small networks of less than 45 nodes because larger networks require prohibitively long computation times $[9,21]$.

Here, we study the transient behavior of networks showing chimera dynamics in an experimental network of Boolean phase oscillators realized with electronic logic circuits. Because these nodes operate on a time scale of $\sim 100 \mathrm{~ns}$, we can study the scaling of the transient in large networks of more than a hundred nodes. The transient includes chimera states for about $14 \%$ of the time for $N=128$ and ends in a nearly synchronized state. We find that the transient time follows a Poisson process with an average transient time that increases exponentially with the network size, which is a result of the synchronization rate that follows a power law of the phase-space volume.
The oscillatory network nodes are realized with unclocked logic circuits and directly wired links on microelectronic chips, realizing an autonomous Boolean network $(\mathrm{ABN})$. Besides their application as engineered systems for random number generation and neuromorphic computation [22-24], ABNs are also a common model for genetic circuits [25-27]. The $\mathrm{ABN}$ studied here is a variant of all-digital phase-locked loops, which are widely used for frequency synthesis [28,29].

We study networks of $N$ coupled Boolean phase oscillators as shown schematically in Fig. 1(a), where oscillator $i \in$ $\{1,2, \ldots, N\}$ is nonlocally coupled to multiple other oscillators $j$ forming a network. The oscillators consist of an inverter gate with delayed feedback as shown in Fig. 1(b). For constant delay $\tau_{i}$, this setup is known as a ring oscillator with a frequency given by [30]

$$
f_{i}=\frac{1}{2 \tau_{i}},
$$

where the factor 2 accounts for inverted delayed feedback (one period includes two inversions). We extend the oscillator to allow for an adjustable frequency by making the delay $\tau_{i}$ state dependent so that $\tau_{i}$ and $f_{i}$ change in response to the coupling signals. The coupling signals are generated by measuring the phase difference between the local oscillator and its neighbors, as introduced in Ref. [31] for two oscillators.

The state-dependent delay $\tau_{i}$ of an oscillator is built from unclocked logic gates as shown in Fig. 1(c). It includes a constant delay $\tau_{0, i}[31]$, and a variable delay realized with a combination of XOR logic gates, Boolean switches, and short constant delay lines $\sigma_{i}$. The XOR logic gates generate a signal $x_{i} \oplus x_{j}$ that approximates the phase difference between the $i$ th oscillator $\left(x_{i}\right)$ and its $j$ th neighbor oscillator $\left(x_{j}\right)$. This signal activates one of two paths in the setup of which one has an additional constant delay. When all phase differences are zero $\left(x_{i} \oplus x_{j}=0\right.$ for all $\left.j\right)$, then the maximum feedback delay is selected with $\tau_{i}=\tau_{0, i}+2 R \sigma_{i}$. When, on the other hand, a phase difference is detected $\left(x_{i} \oplus x_{j}=1\right)$, the delay decreases by $\sigma_{i}$. This behavior can be expressed with state-dependent 
(a)

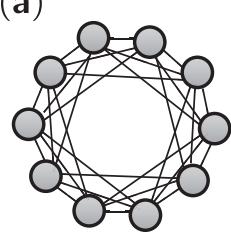

(b)

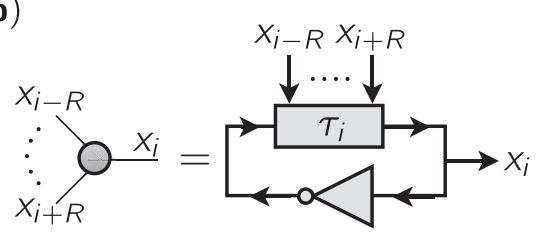

(c)

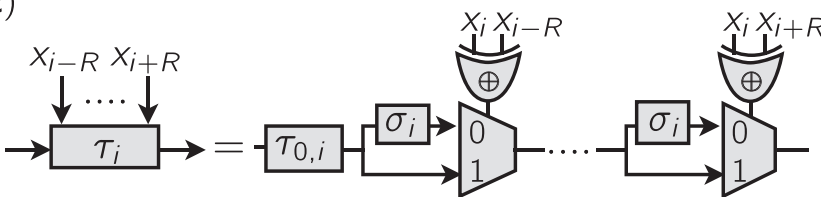

FIG. 1. (a) Illustration of a ring network with $N=10$ nodes and coupling range $R=3$. (b) Illustration of the Boolean phase oscillator (a node in the network) with state variable $x_{i}$. (c) The state-dependent delay for the coupling mechanism consisting of a constant delay $\tau_{0, i}$ built with 30 cascaded copier logic gates and $2 R$ variable delay elements. $\sigma_{i}$, trapezoids, and $\oplus$ signs denote delay lines, Boolean switches (multiplexers), and XOR gates, respectively.

delay

$$
\tau_{i}=\tau_{0, i}+\sigma_{i} \sum_{\substack{j=i-R \\ j \neq i}}^{i+R}\left(1-x_{i} \oplus x_{j}\right),
$$

which is inserted in Eq. (1) to approximate the frequency adjustment, leading to a coupling mechanism of oscillators and hence the possibility of synchronization [31].

As detailed in the Supplemental Material [32], combining Eqs. (1) and (2) leads to an approximate phase model for the Boolean phase oscillators

$$
\dot{\phi}_{i}=\omega_{0, i}+\tilde{\sigma}_{i} \sum_{j=i-R}^{i+R}\left|\Theta\left[\sin \left(\phi_{j}\right)\right]-\Theta\left[\sin \left(\phi_{i}+\alpha_{i j}\right)\right]\right|,
$$

with free-running frequencies $\omega_{0, i}$, coupling strengths $\tilde{\sigma}_{i}$, phase lag parameter $\alpha_{i j}$ that results from transmission delays, and Heaviside function $\Theta$. The oscillators are nonlocally coupled in a ring network with a coupling range $R$ as shown schematically in Fig. 1(a). This configuration has been used previously to observe chimera states with the Kuramoto model, which is similar to Eq. (3) $[1,2,4-7,9,33]$.

The experimental oscillators have an intrinsic frequency heterogeneity of $\left|\sigma_{f}\right| / \bar{f}=0.3 \%$ with average frequency $\bar{f}=$ $9.14 \mathrm{MHz}$ and standard deviation $\sigma_{f}=0.03 \mathrm{MHz}$ [32]. In the model, we assume identical oscillators $\left(\omega_{0, i}=\omega_{0}\right)$ and homogeneous coupling $\left(\tilde{\sigma}_{i}=\tilde{\sigma}\right.$ and $\left.\alpha_{i j}=\alpha\right)$.

We first describe a part of the network dynamics in Fig. 2(a), showing a snapshot of the phase of oscillators in a chimera state. The oscillators outside (inside) the dotted lines, marked region I (region II), have equal (different) phases within our measurement precision of $\Delta \phi= \pm 0.25 \mathrm{rad}$ and hence are considered phase synchronized (desynchronized). Therefore, the oscillators in region I stay synchronized, whereas those in region II drift apart because they have different frequencies. These frequencies are shown in Fig. 2(b) and are measured over a time period of $6 \mu \mathrm{s}$, which represents approximately 60 oscillation periods with precision of $\pm 0.2 \mathrm{MHz}$. The

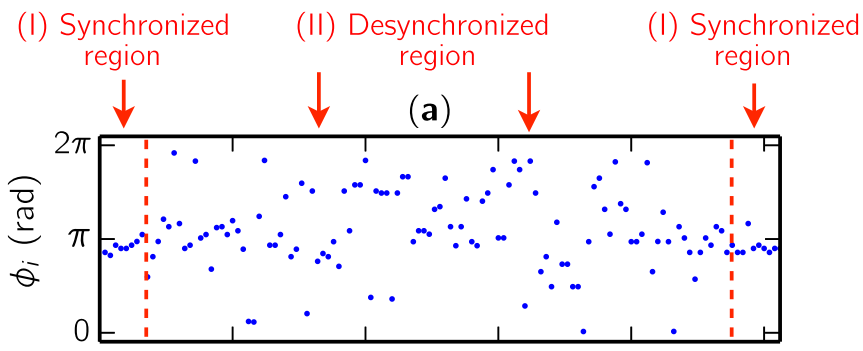

(b)

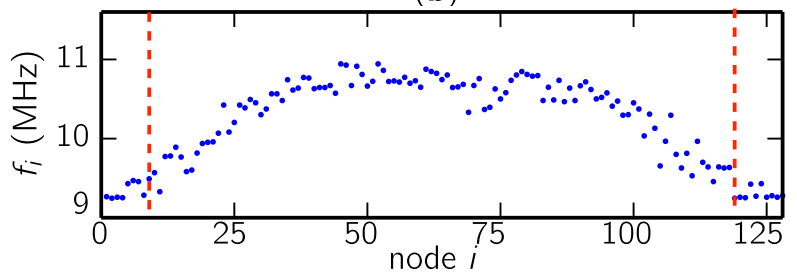

FIG. 2. (Color online) Dynamics measured from coupled Boolean phase oscillators with $N=128, R=30, \omega_{0}=2 \pi(9.3 \pm$ $0.03) \mathrm{MHz}, \quad \tilde{\sigma}=2 \pi(0.089 \pm 0.003) \mathrm{MHz}$. (a) Snapshot at $t \approx 304 \mathrm{~s}$; (b) frequency profile $f_{i}=\left\langle\dot{\phi}_{i}\right\rangle /(2 \pi)$. The network is initialized by deactivating the coupling, resulting in randomized initial phases, followed by activating the coupling. $i$ is shifted by a constant to center the unsynchronized domain [32].

oscillators in region II show the characteristic spectral feature of chimera states [1,2].

The temporal evolution of the frequency is visualized in Fig. 3(a) for a duration of $\sim 7$ min, corresponding to $\sim 4$ billion periods. For this specific realization, complex dynamics exists from time $t=0$ until $t=6$ min (marked III), where the frequency varies both from node to node and in time. At time $t=6 \mathrm{~min}$, the dynamics collapses to a nearly synchronized state (dark gray region, marked IV), where all but $\leqslant 10$ oscillators have a frequency of $f=11.085 \pm 0.002 \mathrm{MHz}$ (compare to $f=9.14 \pm 0.03 \mathrm{MHz}$ for uncoupled oscillators). The remaining oscillators have a frequency different from the synchronized frequency by about $1 \%$ because of heterogeneity [32]. The time until synchronization varies considerably for different experimental runs.

In the following, we discuss the dynamics on a microsecond time scale, at times marked in Fig. 3(a).

Figure 3(b) shows the frequency of the oscillators for about 60 periods after $304 \mathrm{~s}$, corresponding to a millionth of the total transient. The network shows high frequencies (dark gray) for oscillator indices from $i \cong 20$ to $i \cong 100$ and low frequencies (light gray) for the remaining oscillators. This figure corresponds to the chimera state already identified in Fig. 2(a). The unsynchronized domain of the chimera state (high frequency, dark gray) moves irregularly in the network because of finite-size effects [4,34]; this also indicates that the chimera state is not pinned to the network heterogeneities.

At an earlier time in the transient shown in Fig. 3(c), the dynamics alternates between complete desynchronization and chimera states. For times $0<t<2.5 \mu \mathrm{s}$ (marked V), the figure shows large variations in the frequencies of neighboring nodes but with no obvious chimera domain (see phase analyses in [32]). In the remaining time interval (marked VI), two domains of high and low frequencies can be identified, which 

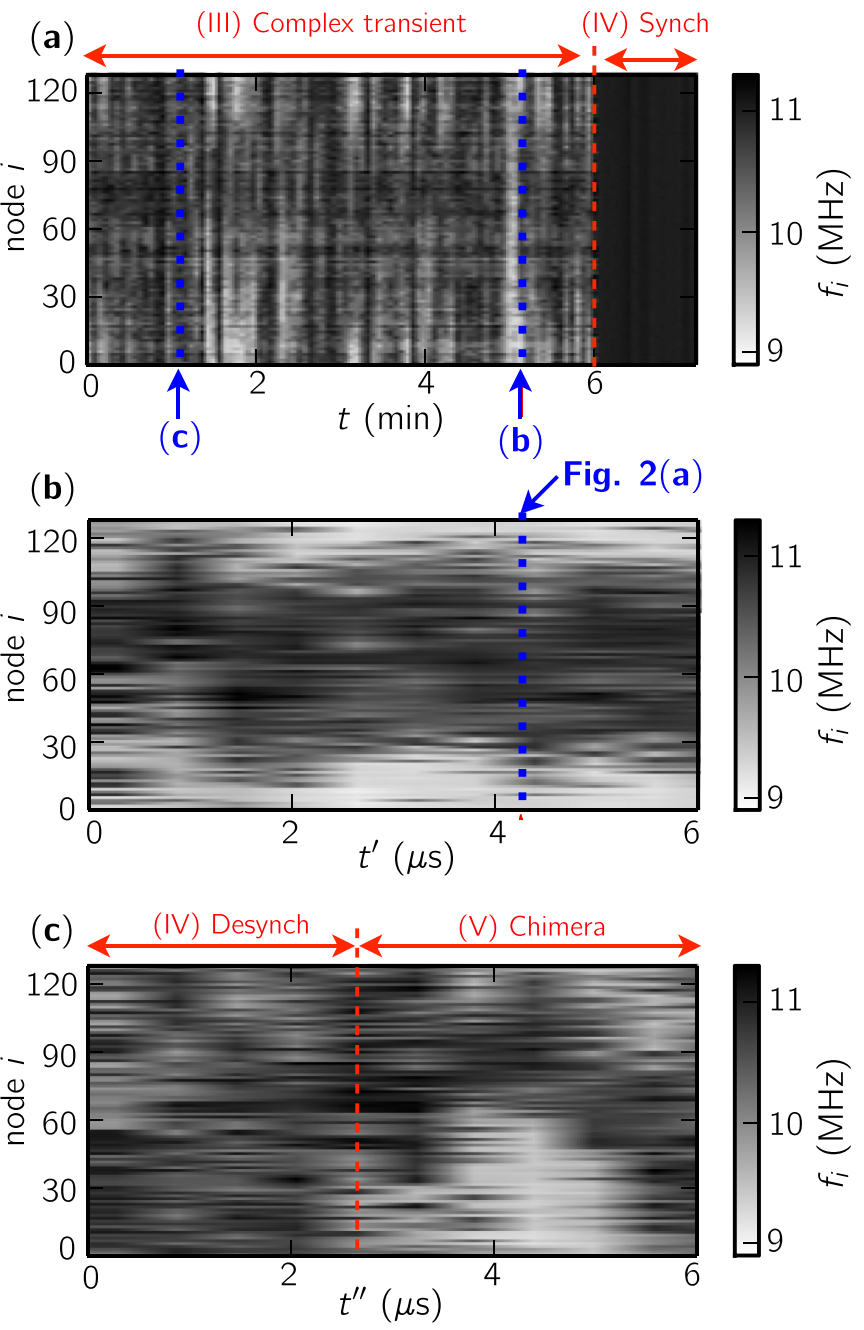

FIG. 3. (Color online) (a) Frequency evolution over a time period of $7 \mathrm{~min}$; averaged over $6 \mu$ s windows (60 oscillations) every $4 \mathrm{~s}$. (b), (c) Frequency evolutions shown over a time period of $5 \mu \mathrm{s}$; averaged over $500 \mathrm{~ns}$ windows (five oscillations) with (b) $t=t^{\prime}+$ $304 \mathrm{~s}$ and (c) $t=t^{\prime \prime}+56 \mathrm{~s}$. The arrow in (b) indicates the phase measurement in Fig. 2(a). Parameters of the experiment as in Fig. 2.

correspond to a chimera state that moves in the network and lasts for $\sim 30$ oscillations. We are the first to report on this reappearance and disappearance of chimera states, which we call resurgence of chimera states.

After a transient time $T_{N}$, the complex dynamics collapses to a synchronized state. We find that $T_{N}$ varies between extreme values of $T_{N}=1 \mathrm{~s}$ and $T_{N}=32 \min$ for $N=128$ and 1000 measurements from different random initializations. Different from Ref. [19], chimeras appear at every acquisition. Figure 4(a) shows the experimental distribution $\rho_{N}$ of transient times, where each dot corresponds to the normalized number of transients with a given lifetime $T_{N}$. We find that $T_{N}$ follows an exponential distribution (solid line) according to

$$
\rho_{N}\left(T_{N}\right)=\left\langle T_{N}\right\rangle^{-1} \exp \left(-T_{N} /\left\langle T_{N}\right\rangle\right),
$$

with the average transient time $\left\langle T_{N}\right\rangle=5.4 \mathrm{~min}$ based on our experimental measurements for $N=128$. The exponential distribution follows analytically by considering the collapse
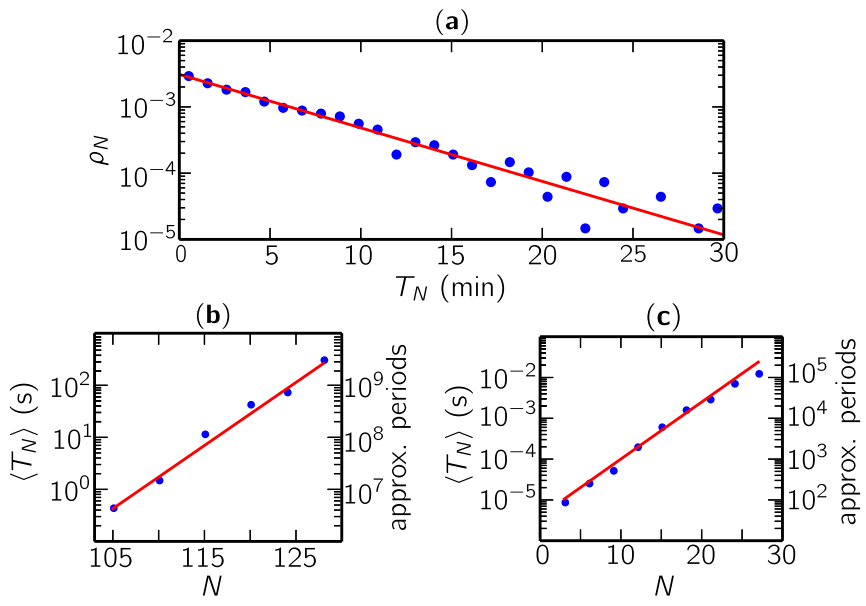

FIG. 4. (Color online) (a) Histogram of transient times $T_{N}$ with $N=128$ from 1000 experimental acquisitions (circles) and distribution function Eq. (4) (solid line). (b), (c) Average transient time $\left\langle T_{N}\right\rangle$ as a function of $N$ measured from (b) 1000 experimental transients each, (c) 200 simulated transients each (circles). Both are fitted with Eq. (5) (solid line) with (b) $\kappa=0.28 \pm 0.10$, (c) $\kappa=0.30 \pm 0.08$. The right axis shows the approximate number of periods per transient. Experimental parameters $R / N \approx 0.24, \omega_{0} \approx 1000 /[19.7+2.9 R]$ (see [32]), and $\tilde{\sigma}=\sigma \omega_{0}^{2} / \pi$ with $\sigma=0.515 \pm 0.018 \mathrm{~ns}$; initial conditions as in Fig. 2. Numerical parameters are $R / N=1 / 3$, $\tilde{\sigma}=0.089 \mathrm{MHz} \times 40 / R, \alpha=0.1$ and initial conditions as in Fig. 5. $N$ in (c) is limited by available computation time.

to synchronization as a Poisson process, which occurs continuously in time at a constant average synchronization rate $\lambda=1 /\left\langle T_{N}\right\rangle$.

Such exponential distribution has been found theoretically to describe the transient times for chimera states in the $\mathrm{Ku}$ ramoto model under the assumption of identical oscillators [9]. The appearance of the same scaling is very interesting because our experiment has heterogeneity and shows resurgence of chimeras, which are not included in previous models.

We measure the average transient time $\left\langle T_{N}\right\rangle$ for networks of different size $N$ and the same network topology. Figure 4(b) shows $\left\langle T_{N}\right\rangle$ for six different network sizes from $N=105$ to $N=128$. The average transient time $\left\langle T_{N}\right\rangle$ follows approximately an exponential scaling over three orders of magnitude according to

$$
\left\langle T_{N}\right\rangle \propto \exp (\kappa N),
$$

with $\kappa=0.28 \pm 0.10$. Using Eq. (5) and the assumption of a Poisson process, the synchronization rate follows $\lambda \propto$ $\exp (-\kappa N) \propto V^{-\kappa}$, which is a power law of the network state-space volume $V=(2 \pi)^{N}$. This is plausible assuming for a single oscillator's phase-space volume $V=2 \pi$ in accordance with Eq. (3). This supertransient scaling holds for many spatially extended systems [11], neural networks [35], and networks of Kuramoto oscillators [9]. This suggests that the synchronization rate $\lambda \propto V^{-\kappa}$ may be a general law for networks under certain conditions, such as nearly identical nodes and the existence of a stable synchronized state.

We study the network dynamics numerically using the simplified model in Eq. (3). Analogous to Fig. 2, Fig. 5 shows the dynamics in phase and frequency representations. We use 


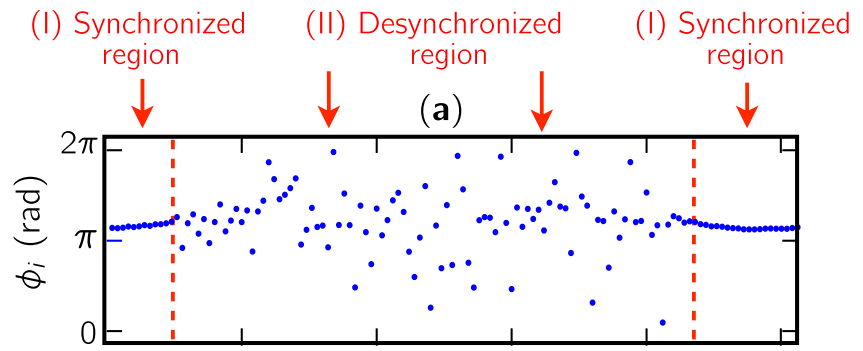

(b)

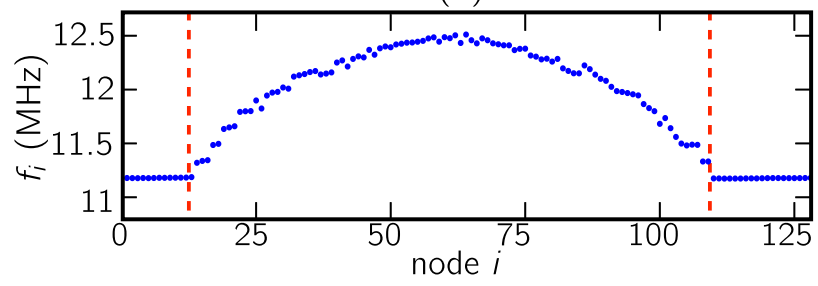

FIG. 5. (Color online) (a) Phases $\phi_{i}$ and (b) frequencies $f_{i}=$ $\left\langle\dot{\phi}_{i}\right\rangle /(2 \pi)$ of the network at $t=50 \mu \mathrm{s}$. Dynamics are obtained from numerical simulation of Eq. (3) with $N=128, R=42$, $\omega_{0}=2 \pi \times 9.3 \mathrm{MHz}, \tilde{\sigma}=2 \pi \times 0.089 \mathrm{MHz}, \alpha=0.1$. Dynamics are initialized as in Ref. [2] with $\phi_{i}=6 p \exp \left(-0.76 x^{2}\right)$, where $p$ is a uniform random variable on $[-0.5,0.5]$ and $x=2 \pi i / N-\pi$. For simplicity, we do not assume frequency heterogeneity and noise in the model. To improve simulation performance, we simulate an altered version of Eq. (3) with a continuous XOR function given by $\left\{\tanh \left[-c \sin \left(\phi_{j}\right) \sin \left(\phi_{i}+\alpha\right)\right]+1\right\} / 2$ with slope $c=4$ instead of $\left|\Theta\left[\sin \left(\phi_{j}\right)\right]-\Theta\left[\sin \left(\phi_{i}+\alpha\right)\right]\right|$.

a different coupling range of $R=42(R / N \approx 1 / 3)$ than in the experiment because the value used in the experiment $(R=30)$ does not lead to chimera states in the simulation. The figure shows a chimera state with coexistence of a synchronized and desynchronized domain (see also the explanation for Fig. 2). The model also reproduces the characteristic scaling of the transient of Eq. (5), as shown in Fig. 4(c) with $\kappa=0.30 \pm$ 0.08 , which is similar to Fig. 4(b). Both results suggest that the model is well suited to describe our experiment qualitatively.

The model is, however, only a first step towards a complete theoretical description of the experimental dynamics because of several differences. First, the simulation shows chimera states for the entire transient and does not show the resurgence of chimeras as in the experiment [32]. Second, the simulation (experiment) collapses to a synchronized (nearly synchronized) state, where nodes are phase and frequency synchronized (nearly frequency synchronized but not phase synchronized) after the transient [32]. Third, chimera states appear in different parameter regions in the model and experiment.

These differences may be caused by heterogeneity in the experiment $\alpha_{i j} \neq$ const, while $\alpha_{i j}=$ const is assumed in the model. Specifically, the experiment implements heterogeneous wiring leading to differences in link delays [32]. Furthermore, differences may be caused by noise and frequency heterogeneity of $0.3 \%$, and transmission delays along the links $(<5 \mathrm{~ns})$ in the experiment. Future work has to fill this gap to uncover the underlying mechanism.

In conclusion, we study a network of Boolean phase oscillators that approximately follows equations similar to the Kuramoto model [1]. Large experimental networks of up to 128 nonlocally coupled Boolean phase oscillators show complex transient dynamics, where chimera states disappear and reappear, called resurgence of chimera states, which is not yet theoretically understood. The dynamics collapses to a synchronized state after a long transient, which can be modeled by a Poisson process with an average lifetime scaling exponentially with the network size, as predicted theoretically in coupled Kuramoto oscillators [9]. The appearance of supertransient scaling in our experimental networks provides further evidence that this scaling could be a general feature of certain networks. Our work motivates future experimental studies, such as transient scaling in spiking neural networks and control of chimera states [36].

The authors gratefully acknowledge financial support by the U.S. Army Research Office within Grant No. W911NF12-1-0099. D.P.R. and E.S. acknowledge the DFG for financial support in the framework of SFB910. D.R. acknowledges Fondation Supélec for financial support. D.P.R. thanks Philipp Hövel for helpful discussions.
[1] Y. Kuramoto and D. Battogtokh, Nonlinear Phenom. Complex Syst. 5, 380 (2002).

[2] D. M. Abrams and S. H. Strogatz, Phys. Rev. Lett. 93, 174102 (2004).

[3] D. M. Abrams, R. Mirollo, S. H. Strogatz, and D. A. Wiley, Phys. Rev. Lett. 101, 084103 (2008).

[4] O. E. Omel'chenko, M. Wolfrum, and Y. L. Maistrenko, Phys. Rev. E 81, 065201(R) (2010).

[5] I. Omelchenko, Y. Maistrenko, P. Hövel, and E. Schöll, Phys. Rev. Lett. 106, 234102 (2011).

[6] A. Zakharova, M. Kapeller, and E. Schöll, Phys. Rev. Lett. 112, 154101 (2014).

[7] I. Omelchenko, O. E. Omel'chenko, P. Hövel, and E. Schöll, Phys. Rev. Lett. 110, 224101 (2013).
[8] M. J. Panaggio and D. M. Abrams, arXiv:1403.6204.

[9] M. Wolfrum and O. E. Omel'chenko, Phys. Rev. E 84, 015201 (2011).

[10] I. Simonsen, L. Buzna, K. Peters, S. Bornholdt, and D. Helbing, Phys. Rev. Lett. 100, 218701 (2008).

[11] T. Tél and Y.-C. Lai, Phys. Rep. 460, 245 (2008).

[12] A. Hastings and K. Higgins, Science 263, 1133 (1994).

[13] R. Rabinovich, R. Huerta, and G. Laurent, Science 321, 48 (2008).

[14] M. Newman, Networks: An Introduction (Oxford University Press, New York, 2010).

[15] A. Hagerstrom, T. E. Murphy, R. Roy, P. Hövel, I. Omelchenko, and E. Schöll, Nat. Phys. 8, 658 (2012). 
[16] M. R. Tinsley, S. Nkomo, and K. Showalter, Nat. Phys. 8, 662 (2012).

[17] E. A. Martens, S. Thutupalli, A. Fourrière, and O. Hallatschek, Proc. Natl. Acad. Sci. U.S.A. 110, 10563 (2013).

[18] L. Larger, B. Penkovsky, and Y. L. Maistrenko, Phys. Rev. Lett. 111, 054103 (2013).

[19] M. Wickramasinghe and I. Z. Kiss, PloS ONE 8, e80586 (2013).

[20] L. Schmidt, K. Schönleber, K. Krischer, and V. Garcia-Morales, Chaos 24, 013102 (2014).

[21] A. E. Motter, M. Gruiz, G. Károlyi, and T. Tél, Phys. Rev. Lett. 111, 194101 (2013).

[22] D. P. Rosin, D. Rontani, D. J. Gauthier, and E. Schöll, Phys. Rev. Lett. 110, 104102 (2013).

[23] D. P. Rosin, D. Rontani, and D. J. Gauthier, Phys. Rev. E 87, 040902(R) (2013).

[24] D. P. Rosin, D. Rontani, D. J. Gauthier, and E. Schöll, Chaos 23, 025102 (2013).

[25] S. A. Kauffman, J. Theoret. Biol. 22, 437 (1969).
[26] M. Ghil and A. Mullhaupt, J. Stat. Phys. 41, 125 (1985).

[27] L. Glass and C. Hill, Europhys. Lett. 41, 599 (1998).

[28] S. R. Al-araji, Z. M. Hussain, and M. A. Al-qutayri, Digital Phase Lock Loops (Springer Verlag, Dordrecht, Netherland, 2006).

[29] R. E. Best, Phase-Locked Loops (McGraw-Hill, New York, 2003).

[30] H. Kato, IEEE Trans. Circuits Syst. I 45, 98 (1998).

[31] D. P. Rosin, D. Rontani, and D. J. Gauthier, Phys. Rev. E 89, 042907 (2014).

[32] See Supplemental Material at http://link.aps.org/supplemental/ 10.1103/PhysRevE.90.030902 for further information.

[33] Y. Kuramoto, Chemical Oscillations, Waves, and Turbulence (Springer, New York, 1984).

[34] S. Nkomo, M. R. Tinsley, and K. Showalter, Phys. Rev. Lett. 110, 244102 (2013).

[35] R. Zillmer, N. Brunel, and D. Hansel, Phys. Rev. E 79, 031909 (2009).

[36] J. Sieber, O. E. Omel'chenko, and M. Wolfrum, Phys. Rev. Lett. 112, 054102 (2014). 


\title{
Supplementary material for 'Transient scaling and resurgence of chimera states in coupled Boolean phase oscillators'
}

\author{
David P. Rosin, ${ }^{1,2}$ Damien Rontani, ${ }^{1,3}$, Nicholas D. \\ Haynes, ${ }^{1}$ Eckehard Schöll, ${ }^{2}$ and Daniel J. Gauthier ${ }^{1}$ \\ ${ }^{1}$ Department of Physics, Duke University, \\ 120 Science Drive, Durham NC 27708, USA \\ ${ }^{2}$ Institut für Theoretische Physik, Technische Universität Berlin, \\ Hardenbergstr. 36, Berlin D-10623, Germany \\ ${ }^{3}$ Supélec, OPTEL research group and LMOPS EA-4423, \\ 2 Rue Edouard Belin, Metz F-57070, France
}

(Dated: May 7, 2014) 


\section{DERIVATION OF A PHASE MODEL FOR BOOLEAN PHASE OSCILLATORS}

In this section, we derive a phase model for Boolean phase oscillators shown in the setup in Fig. 1(c) in the main article. The oscillators are described by output variables $\left\{x_{i}\right\}_{i=1}^{N}$, which are normalized output voltages $\left\{V_{i}\right\}_{i=1}^{N}$ of oscillators so that $x_{i}=1\left(x_{i}=-1\right)$ corresponds to the high (low) Boolean voltage; specifically $x_{i}=2 V_{i} / V_{H}-1$ with high Boolean voltage $V_{H}$ [1]. The coupling of oscillators is realized with state-dependent delay that depends on the phase differences between the oscillator output $x_{i}$ and $K$ oscillatory input signals $\left\{x_{j i}^{\text {in }}\right\}$. Delay lines in the setup are based on chains of buffer gates that each add a gate propagation delay to the total delay as detailed in Ref. [1]. In the setup in Fig. 1(c), we include several delay lines of value $\sigma=0.328 \pm 0.012 \mathrm{~ns}$, corresponding to a single buffer gate and a long delay line of value $\tau=8.4 \pm 0.3 \mathrm{~ns}$, corresponding to 30 cascaded buffer gates. Several XOR logic gates are included as simple Boolean phase detectors [2] to generate a control signal $x_{j i}^{c} \in\{0,1\}$. The control signal is given by a comparison of the output Boolean state of the oscillator $x_{i}$ and an input Boolean state $x_{j i}^{\text {in }}$, according to

$$
x_{j i}^{c}=x_{j i}^{\mathrm{in}} \oplus x_{i},
$$

where $\oplus:\{0,1\} \times\{0,1\} \rightarrow\{0,1\}$ denotes the XOR Boolean function. Several multiplexers, which are 3-input logic gates, are included as Boolean switches to modify the feedback delay of the system by a delay $\sigma_{i}$ depending on the control signals $\left\{x_{j i}^{c}\right\}$. This leads to the total feedback delay of the $i$-th oscillator according to

$$
\tau_{i}=\tau_{m, i}-\sigma_{i} \sum_{j=0}^{K} x_{j i}^{c},
$$

where $\tau_{m, i}=\tau_{0, i}+K \sigma_{i}$ denotes the maximum delay in the loop. This is a state-dependent delay because the $\left\{x_{j i}^{c}\right\}_{j}$ depend on the state of the local oscillator [3].

We also include a single inverter logic gate in the feedback loop in Fig. 1(c) that is known to lead to stable oscillations due to negative delayed feedback similar to a ring oscillator [4]. The resulting frequency of the $i$-th oscillator is, under the assumption of a constant feedback delay,

$$
f_{i}=\frac{1}{2 \tau_{i}} .
$$

Here the factor 2 comes in due to inverted delayed feedback, where two round trips lead to the initial state. 
We assume that Eq. (S3) is valid for state-dependent delay when the frequency $f_{i}$ is replaced with the instantaneous frequency $\dot{\phi}_{i}$. Using Eqs. (S1)-(S3) and a Taylor approximation with a maximum error of $\approx 3 \mathrm{MHz}$ for our parameters, we find

$$
\dot{\phi}_{i}=\omega_{0, i}+\tilde{\sigma}_{i} \sum_{j=j_{0}}^{j_{K}} x_{j}\left(\phi_{j}\right) \oplus x_{i}\left(\phi_{i}\right)
$$

with angular frequency $\omega_{0, i}=2 \pi /\left(2 \tau_{0, i}\right)=2 \pi \cdot 9.3 \mathrm{MHz}$ (for in-degree $K=60$ ) and coupling strength $\tilde{\sigma}_{i}=2 \sigma \omega_{0, i}^{2}=0.089 \mathrm{MHz}$ (using a value $\sigma=0.515 \mathrm{~ns}$ to adjust for the error by the Taylor approximation). The summation from $j_{0}$ to $j_{K}$ is over $K$ coupling inputs corresponding to the non-local network topology. We replace $\left\{x_{j}\right\}$ with $\left\{\phi_{j}\right\}$ using the following definition of the phase $\phi_{j}$ from Ref. [5]

$$
\sin \left[\phi_{j}(t)\right]=\frac{2 x_{j}(t)-\max \left[x_{j}\right]-\min \left[x_{j}\right]}{\max \left[x_{j}\right]-\min \left[x_{j}\right]}=x_{j}(t)
$$

where $x_{j}$ oscillates between the Boolean values -1 and 1 . We express the XOR Boolean function with a difference of Heaviside functions and add a phase-lag parameter $\alpha_{i j}$, leading to the following phase oscillator model

$$
\dot{\phi}_{i}=\omega_{0, i}+\tilde{\sigma}_{i} \sum_{j=j_{0}}^{j_{K}}\left|\Theta\left[\sin \left(\phi_{j}\right)\right]-\Theta\left[\sin \left(\phi_{i}+\alpha_{i j}\right)\right]\right|,
$$

The phase-lag parameter $\alpha_{i j}$ is vital for observing chimera states [6, 7] and is likely caused experimentally by delays along wire connections.

We couple the Boolean oscillators in a ring network with non-local coupling realized with a coupling radius $R$, corresponding to a rectangular coupling kernel. Specifically, the resulting dynamical equation for the network is

$$
\dot{\phi}_{i}=\omega_{0, i}+\tilde{\sigma}_{i} \sum_{j=i-R}^{i+R}\left|\Theta\left[\sin \left(\phi_{j}\right)\right]-\Theta\left[\sin \left(\phi_{i}+\alpha_{i j}\right)\right]\right|,
$$

where we consider a network of $N$ nodes $(i \in\{1,2, \ldots, N\})$, and periodic boundary conditions. The free-running frequency of an oscillator $\omega_{0, i}$ as a function of $R$ results from the construction of the oscillator with logic gates as shown in Fig 1(c) in the main article. It is given by $\omega_{0, i}=1 /\left[2\left(30 \tau_{\text {buf }}+2 R \tau_{\text {mux }}+2 R \tau_{\text {buf }}\right)\right]$ with $n_{0}=30$ the number of buffer gates used to build the delay line denoted $\tau$, and the propagation delays of multiplexer and buffer are $\tau_{\text {mux }}=0.404 \pm 0.014 \mathrm{~ns}, \tau_{\text {buf }}=0.328 \pm 0.012 \mathrm{~ns}$, respectively. 


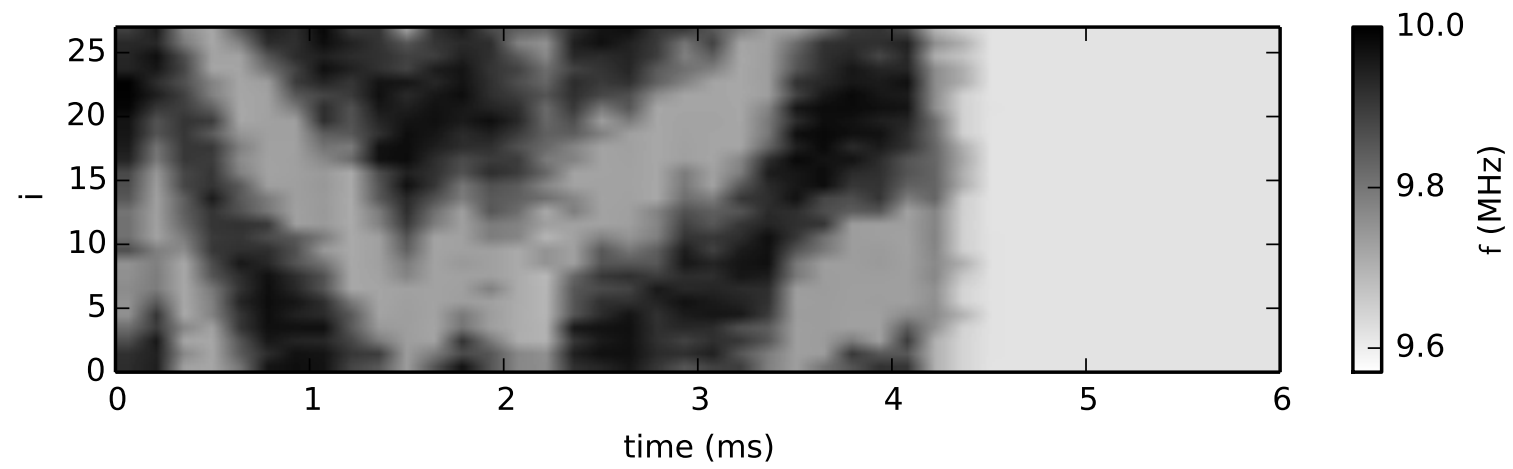

FIG. S1: Frequency evolution from numerical simulation of Fig. S8 with parameters $N=27$, others as in Fig. 4(c) in the main article. Initial condition and approximation as Fig. 5 in the main article.

For numerical simulation, it is advantageous to replace the Heaviside functions and the absolute value in Eq. S7) with a smooth tanh, leading to

$$
\dot{\phi}_{i}=\omega_{0, i}+\tilde{\sigma}_{i} \sum_{j=i-R}^{i+R}\left\{\tanh \left[-c \sin \left(\phi_{j}\right) \sin \left(\phi_{i}+\alpha\right)\right]+1\right\} / 2
$$

with slope $c=4$ used in the numerical simulation.

The numerical simulation of Eq. (S8) leads to a phase and frequency profile (snapshot) shown and discussed in Fig. 5 of the main article. Here, we also discuss the temporal evolution of the frequency that is shown in Fig. S1. Similar to Fig. 3(b) in the main article, the system shows a chimera state that wanders around the network (compare also the corresponding explanation in the main text). After a certain time that depends heavily on the initial conditions, the chimera state collapses to a synchronized state, which happens after $4.2 \mathrm{~ms}$ in Fig. S1.

Note that chimera states appear with Eq. (S7) for phase lag parameters in the vicinity of $\alpha_{i j}=0.1$ in the model, which is different from the common value of $\alpha=\pi / 2-0.18$ used by Abrams and Strogatz to achieve chimera states [7]. This may be due to the differences in the coupling function of the two models, cf. [8], where a systematic phase reduction has been performed. Note also that the experiment does not include self-coupling, but it is common to assume self-coupling in previous numerical studies of Kuramoto networks. 


\section{PHASE SNAPSHOTS FOR FIG. 3(C) OF THE MAIN ARTICLE}

In this section, we discuss the experimentally measured temporal evolution of frequencies and phases in Fig. 3(c) of the main article, which we have identified as complex dynamics that alternates between chimera states and desynchronization. For the reader's convenience, we show the same illustration in Fig. S2 (a) with eight time points marked as (b)-(i), corresponding to the phase snapshots in Fig. S2(b)-(i), respectively. In the first snapshot in Fig. S2(b), there exists a region, where the oscillators have the same phase to within the experimental phase resolution of $\Delta \phi=0.25$. Outside the dotted lines, the oscillators are not phase-synchronized. Therefore, this state corresponds to both synchronization and desynchronization, which is a chimera state. This state exists for six periods, where the synchronized region drifts considerably in the network. In Fig. S2(c), we find an intermediate state, where the phases of the oscillators are distributed randomly so that we cannot identify a phase-synchronized region; hence, it is an unsynchronized state. In Fig. S2(d), a chimeralike state is reached again, with similar properties to the phase snapshot in Fig. S2(b). Therefore, the dynamics change within a timescale similar to the oscillation period from chimera-like states to unsynchronized states and back.

Figure S2(e) shows the phases of the oscillators in the network at a time when two regions (marked with dotted lines) show the same phases within the measurement error. Outside the two regions, the oscillators show large differences in the phase and are hence unsynchronized. This state corresponds to a chimera state of two synchronized and two desynchronized regions, which has been reported before with numerical simulations [8-11]. Similar to the findings in these references, the two synchronized cores are phase shifted by $\pi$.

Figures $\mathrm{S2}(\mathrm{f})$-(i) show the phases of oscillators in the network in the region called VI in the main article. The synchronized region of the chimera state moves from the range $i \cong 75$ to $i \cong 120$ to the range $i \cong 115$ to $i \cong 20$ (periodic). In Fig. S2(h), one oscillator in the synchronized region does not synchronize, which is non-ideal. 
(a)

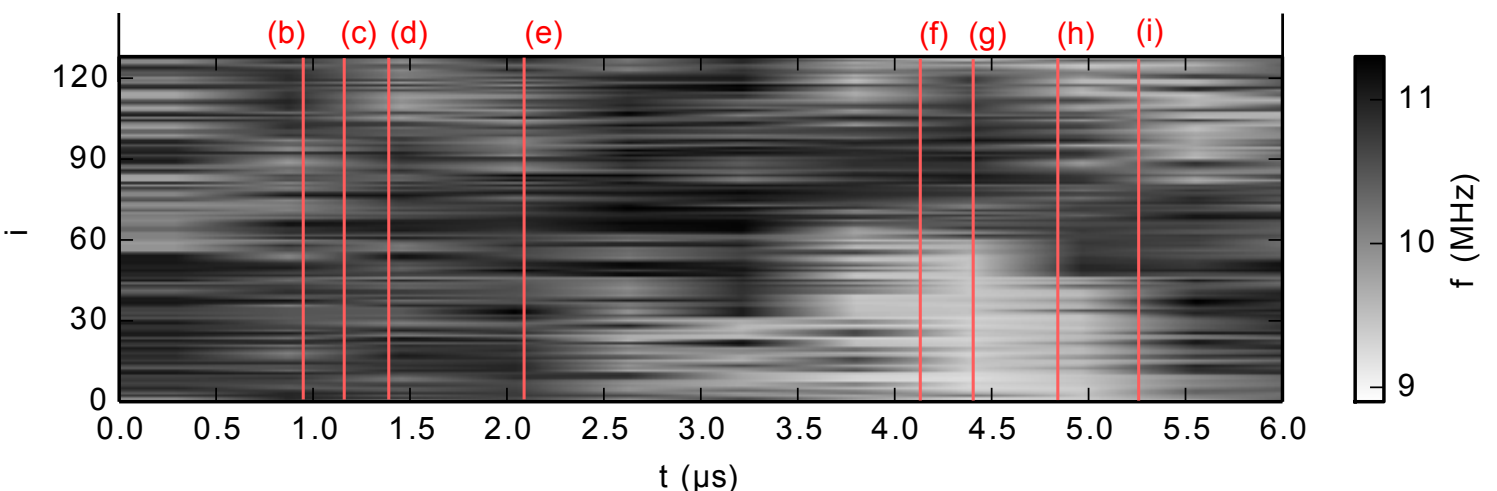

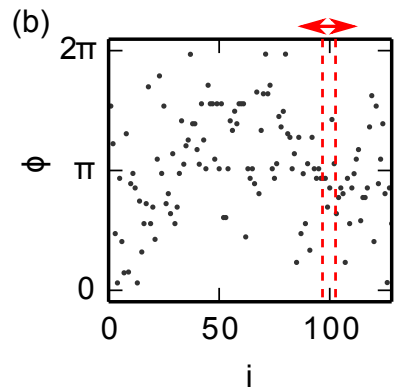

(f)

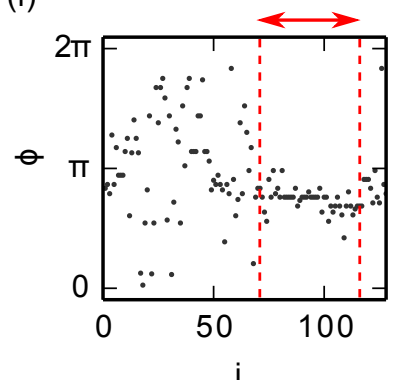

(c)

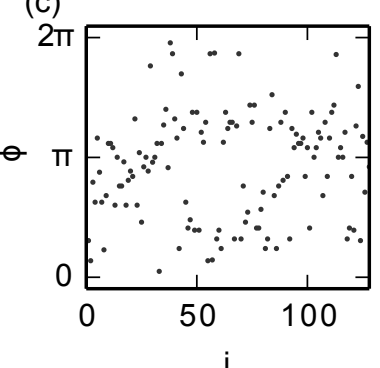

(g)

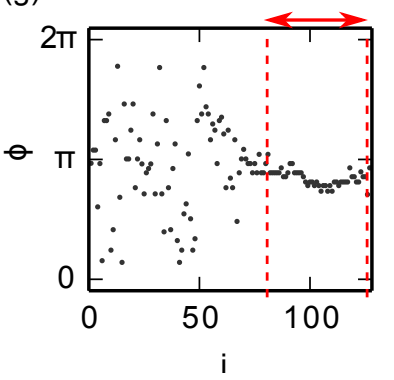

(d)

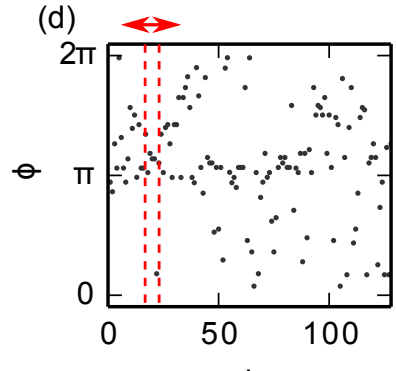

(h)

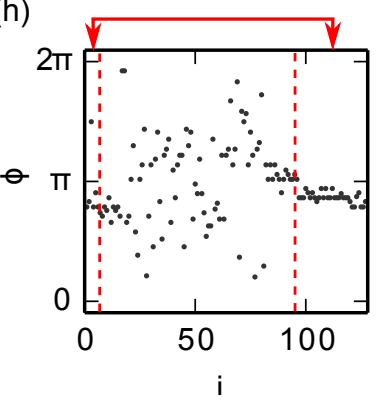

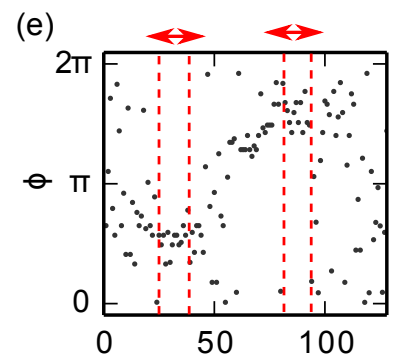

(i)

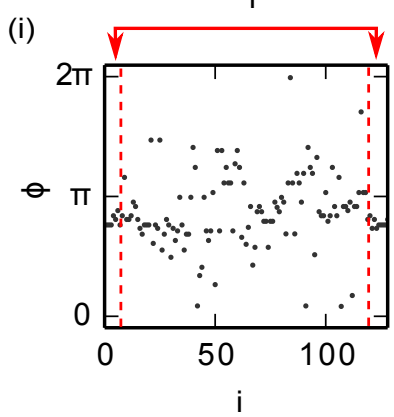

FIG. S2: (a) Temporal evolution of the frequency averaged over $500 \mathrm{~ns}$ windows in the experiment, copied from Fig. 3(c) in the main article. Dotted lines mark the phase pictures shown in (b)(i) at times $0.94 \mu \mathrm{s}, 1.14 \mu \mathrm{s}, 1.37 \mu \mathrm{s}, 2.05 \mu \mathrm{s}, 4.10 \mu \mathrm{s}, 4.42 \mu \mathrm{s}, 4.84 \mu \mathrm{s}$, and $5.26 \mu \mathrm{s}$, respectively. Experimental parameters as in Fig. 3 in the main article.

\section{THE NEARLY SYNCHRONIZED STATE IN SIMULATION AND EXPERI- MENT}

In the main article, we find that the network synchronizes at high frequencies in the experiment, but at low frequencies, equal to the natural frequency, in the model [compare Figs. 3(a) in the main article and Fig. S1. To get more insight into the synchronized state, we show the frequency and phase profiles in Fig. S3. We find that in the experiment, the phases are not synchronized and the frequencies are synchronized apart from two nodes that 

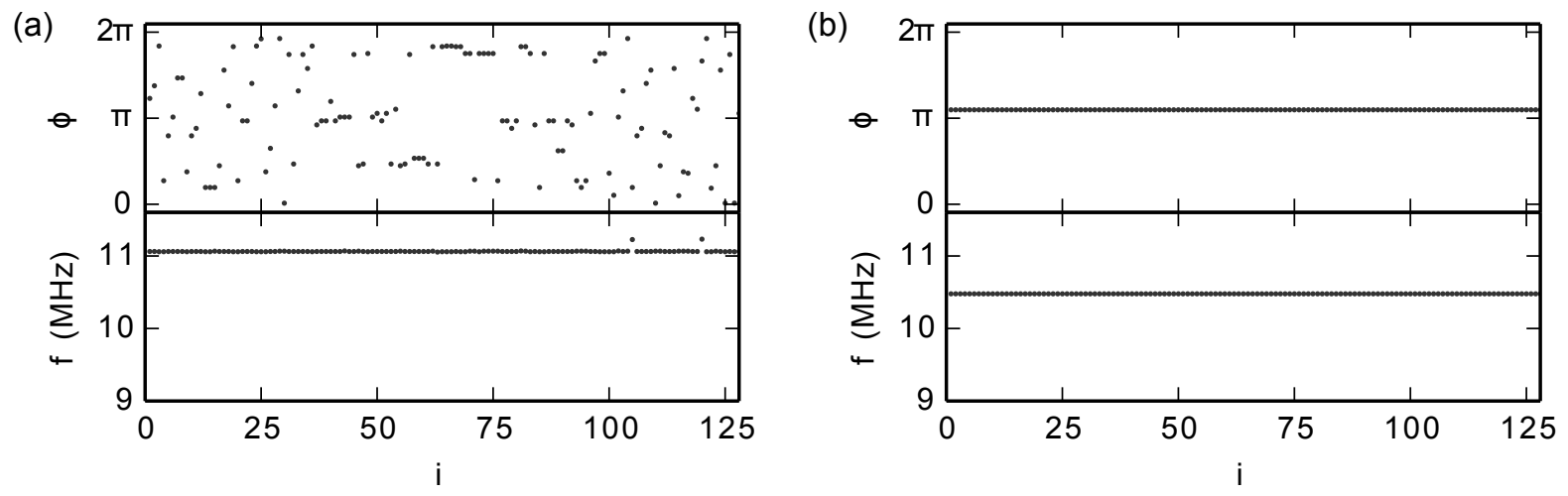

FIG. S3: Snapshots of the synchronized state in (a) experiment and (b) numerical simulation of Eq. S8 with $\alpha_{i, j}=0.1=$ const at (a) $t=480 \mathrm{~s}$ in transient of Fig. 3(a) in the main article and at (b) $t=36 \mu \mathrm{s}$. The simulation was initialized in the synchronized state, but we confirmed with $N=30$ that this state is also reached after a transient via chimera states. Other parameters as in Fig. 5 in the main article.

have $\sim 1 \%$ higher frequency. In the model, on the other hand, both frequency and phase synchronize.

This difference between experiment and model could be, as discussed in the main text, due to heterogeneity in the phase lag parameter $\alpha_{i j}$ in the experiment. When heterogeneity is included in our model in Eq. (S7), however, the synchronized state is still almost synchronized with frequency differences below $0.01 \%$ for heterogeneity $\sigma_{\alpha}<0.2\left(\alpha_{i, j}=0.1 \pm \sigma_{\alpha}\right.$ according to a Gaussian distribution with standard deviation $\sigma_{\alpha}$ ), while, for $\sigma_{\alpha} \geq 0.2$, a stable chimera state is found.

\section{DETERMINING THE FRACTION OF CHIMERA STATES IN THE TRAN- SIENT}

As described in the main article, the transient dynamics includes chimera dynamics among other, more complex dynamics, with chimera-like states that exist only for a few oscillation periods alternating with an unsynchronized state. Here, we determine the fraction of chimera states in the transient with a measure similar to Ref. [12].

The chimera states that we observe in this study have a typical arch shape, where one domain of adjacent oscillators are synchronized at low frequency, and another domain is 
unsynchronized with higher frequencies as shown in Fig. 2(b) of the main article. Such a shape can be detected by measuring the standard deviation of frequencies

$$
\sigma=\sqrt{\left\langle\left(f_{i}-\left\langle f_{i}\right\rangle\right)^{2}\right\rangle}
$$

and the average frequency difference between adjacent oscillators

$$
\Delta=\left\langle\left|f_{i}-f_{i-1}\right|\right\rangle
$$

An arch-shaped function has a large $\sigma$ but a small $\Delta$, so that a large

$$
\psi=\sigma / \Delta
$$

is a measure to detect chimera states.

We calculate this function for the partly synchronized temporal evolution in Fig. 3(c) in the main article, which we have copied for easier comparison in Fig. S4(a). Figure S4(b) shows $\psi$ for this temporal evolution, exhibiting a peak above a value of $\psi=1.5$ approximately when we detect chimera states in the main article. Therefore, we use a threshold of $\psi>1.5$ to distinguish chimera states. With this numeric value, we find that chimera states appear for about $14 \%$ of the transient. This number is obtained from 100 measurements of length $6 \mu$ s on a network of $N=128$ oscillators within the transient shown in Fig. 3(a) of the main article.

\section{TECHNICAL DETAILS OF THE IMPLEMENTATION OF OSCILLATOR NETWORKS ON ELECTRONIC CHIPS}

The experiments in the main article are realized with unclocked circuits of logic gates on the field-programmable gate array (FPGA) Altera Cyclone IV EP4CE115F29C7. The chip is separated into 7155 logic array blocks of 16 programmable logic elements of 4-inputs each. A Boolean phase oscillator is realized with up to 218 logic elements (depending on the frequency adjustment to reduce heterogeneity), requiring 14 logic array blocks. The layout of the oscillators on the chip is shown in Fig. S5. Allocated logic array blocks are marked in darker blue while unused logic array blocks are marked in light blue. The blue rectangle marks a region reserved for the dynamical network. The orange rectangle marks a region reserved for the readout and the acquisition of the network dynamics. About $25 \%$ of the resources on the FPGA are used for the largest implemented network of $N=128$ nodes. 


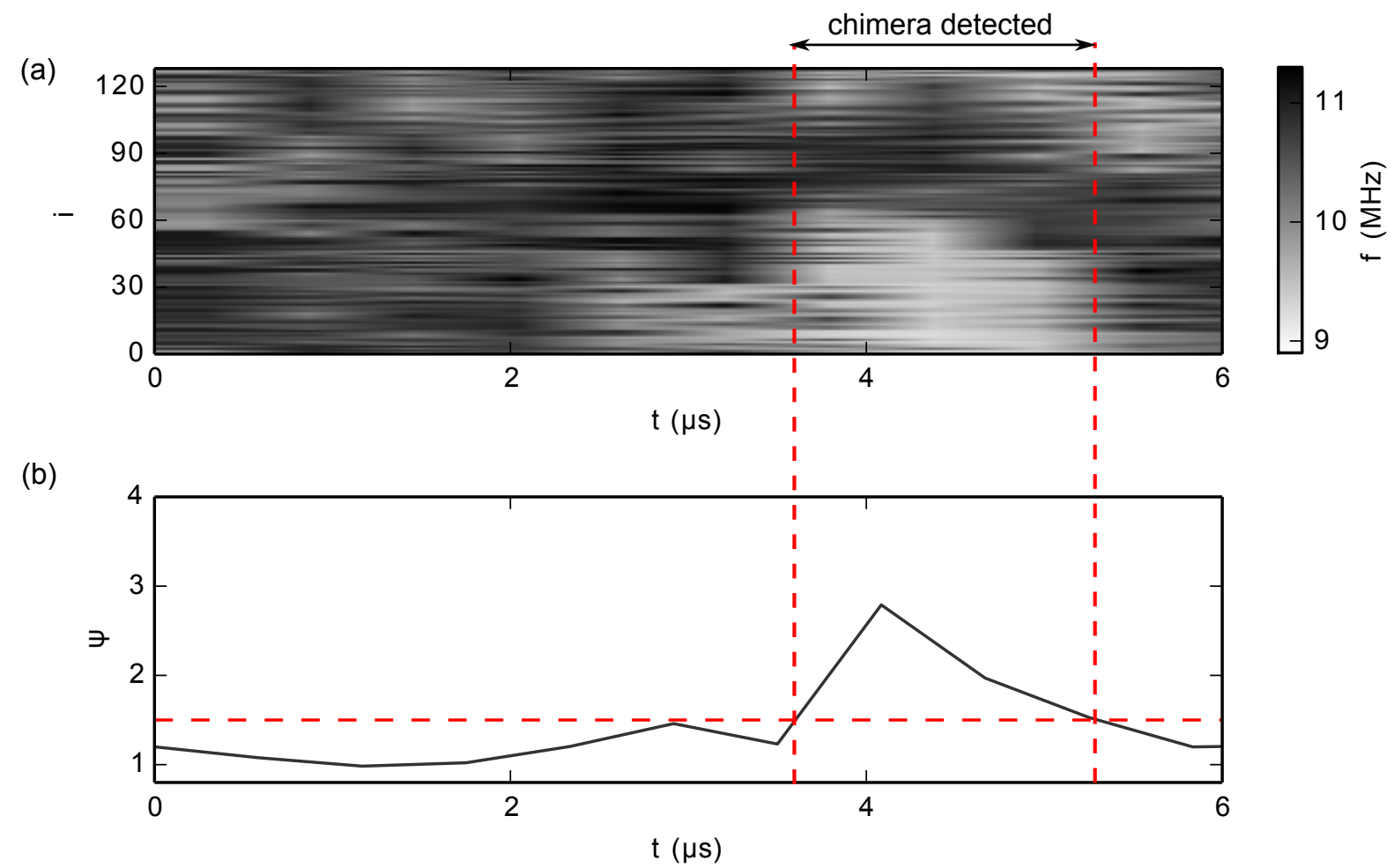

FIG. S4: (a) Copy of Fig. 3(c) in the main article. (b) Corresponding function $\psi$ to detect a chimera state. Specifically, a chimera is detected from time $3.6 \mu$ s until $5.3 \mu$ s when $\psi>1.5$ (shown with dashed lines).

This implementation does not account for the ring structure of the network, so that the delay between some neighboring oscillators, e.g., 18 and 19 and especially 1 and 128, is long compared to other neighbors, such as 1 and 2. We estimate the maximum transmission delay between two oscillators to be 2 ns corresponding to the transmission delay across the chip, which is small compared to the period of about $100 \mathrm{~ns}$.

In addition, the experiment implements heterogeneous wiring, where the $j$-th input $x_{j i}^{\text {in }}$ of node $i$ [Fig. 1(c) in the main article] is given by the $j$-th element of the sorted version of the list $\{(i-R) \bmod N,(i-R+1) \bmod N, \ldots,(i+R) \bmod N\}$. As a consequence, the phase adjustments are made at different positions in the ring depending on the oscillator index. 


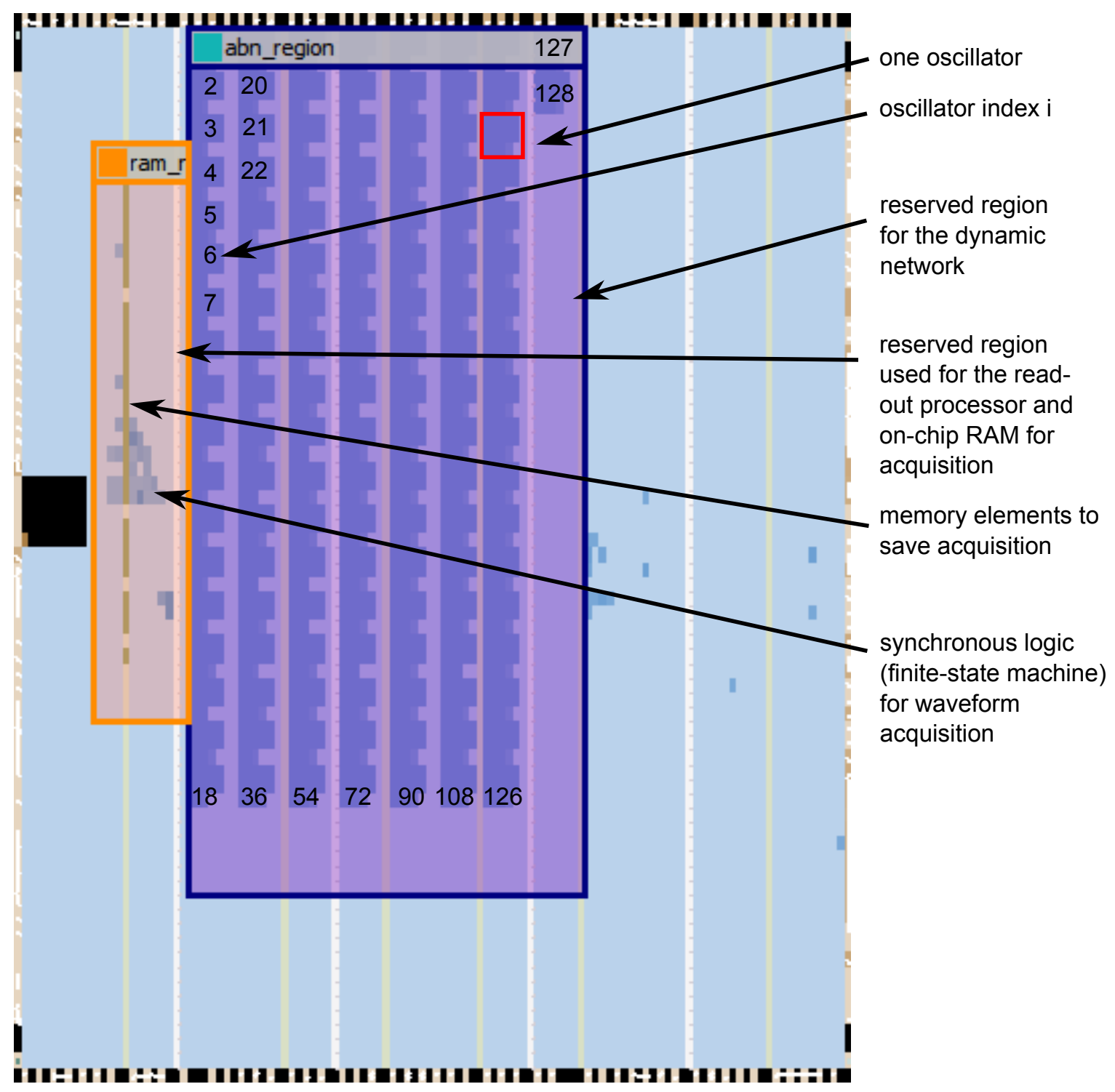

FIG. S5: Picture showing the arrangement of logic gates on the FPGA for the implementation of the network with $N=128$ nodes. The picture was generated with the Altera Chip Planner.

[1] D. P. Rosin, D. Rontani, and D. J. Gauthier, Phys. Rev. E 89, 042907 (2014).

[2] R. E. Best, Phase-Locked Loops (McGraw-Hill, New York, NY, 2003).

[3] H.-O. Walther, J. Diff. Eq. 195, 46 (2003).

[4] H. Kato, IEEE Trans. Circuits Syst. I 45, 98 (1998).

[5] I. Omelchenko, Y. Maistrenko, P. Hövel, and E. Schöll, Phys. Rev. Lett. 106, 234102 (2011). 
[6] Y. Kuramoto and D. Battogtokh, Nonlin. Phen. in Complex Sys. 5, 380 (2002).

[7] D. M. Abrams and S. H. Strogatz, Phys. Rev. Lett. 93, 174102 (2004).

[8] I. Omelchenko, O. E. Omel'chenko, P. Hövel, and E. Schöll, Phys. Rev. Lett. 110, 224101 (2013).

[9] S. Nkomo, M. R. Tinsley, and K. Showalter, Phys. Rev. Lett. 110, 244102 (2013).

[10] S. R. Ujjwal and R. Ramaswamy, Phys. Rev. E 88, 032902 (2013), URL http://link.aps. org/doi/10.1103/PhysRevE.88.032902.

[11] A. Zakharova, M. Kapeller, and E. Schöll, Phys. Rev. Lett. 112, 154101 (2014).

[12] R. R. Gopal, V. K. Chandrasekar, A. Venkatesan, and M. Lakshmanan, Observation and characterization of chimera states in coupled dynamical systems with nonlocal coupling, arXiv:1403.4022 (2014). 